\title{
Comparison Of Some Simple Estimators Of The Lognormal Parameters Based On Censored Samples
}

Baklizi Ayman

YarmoukUniversity, baklizi1@yahoo.com

Mohammed Al-Haj Ebrahem

YarmoukUniversity,m_hassanb@hotmail.com

Follow this and additional works at: http://digitalcommons.wayne.edu/jmasm

Part of the Applied Statistics Commons, Social and Behavioral Sciences Commons, and the Statistical Theory Commons

\section{Recommended Citation}

Ayman, Baklizi and Ebrahem, Mohammed Al-Haj (2006) "Comparison Of Some Simple Estimators Of The Lognormal Parameters Based On Censored Samples," Journal of Modern Applied Statistical Methods: Vol. 5 : Iss. 1 , Article 15.

DOI: $10.22237 /$ jmasm/1146456840

Available at: http://digitalcommons.wayne.edu/jmasm/vol5/iss1/15

This Regular Article is brought to you for free and open access by the Open Access Journals at DigitalCommons@WayneState. It has been accepted for inclusion in Journal of Modern Applied Statistical Methods by an authorized editor of DigitalCommons@WayneState. 


\title{
Comparison Of Some Simple Estimators Of The Lognormal Parameters Based On Censored Samples
}

\author{
Ayman Baklizi Mohammed Al-Haj Ebrahem \\ Department of Statistics \\ Yarmouk University
}

Point estimation of the parameters of the lognormal distribution with censored data is considered. The often employed maximum likelihood estimator does not exist in closed form and iterative methods that require very good starting points are needed. In this article, some techniques of finding closed form estimators to this situation are presented and extended. An extensive simulation study is carried out to investigate and compare the performance of these techniques. The results show that some of them are highly efficient as compared with the maximum likelihood estimator.

Keywords: Modified maximum likelihood estimator, least squares estimators, lognormal distribution, mean squared error, Persson Rootzen estimators

\section{Introduction}

Let the random variable $Y$ be normally distributed with mean $\mu$ and variance $\sigma^{2}$. Let $T=e^{Y}$, then $T$ is said to have a lognormal distribution. The probability density function of $T$ is given by (Lawless, 1982);

$$
f(t)=\frac{1}{t \sigma \sqrt{2 \pi}} \exp \left(-\frac{(\ln t-\mu)^{2}}{2 \sigma^{2}}\right), 0<t<\infty .
$$

The many special features of the lognormal distribution together with its relation with the normal distribution have allowed it to be used as

Ayman Baklizi is an Associate Professor of Applied Statistics. His research interests are in accelerated life tests, censored data, nonparametric statistics, and simulation and resampling methods. Email him at baklizi1@yahoo.com. Mohammed Al-Haj is an Assistant Professor. His research interest is in reliability, accelerated life test and nonparametric regression models. Email: m_hassanb@hotmail.com a model in various real life applications. It is used in analyzing biological data (Koch, 1966), and for analyzing data in workplace exposure to contaminants (Lyles \& Kupper, 1996). It is also of importance in modeling lifetimes of products and individuals (Lawless, 1982). Various other motivations and applications of the lognormal distribution can be found in Johnson et al. (1994) and Schneider (1986).

In most life testing experiments, one is faced with censored data (Lawless, 1982) arising from either terminating the experiment at a certain prespecified time (Type 1 censoring) or when a predetermined number of failures occur (Type 2 censoring). Censoring is often employed because of time and cost considerations. However, complications do often arise in inference from censored data and usually likelihood based inference procedures are used. Assume that the data is Type 2 censored, whereby the following is observed: $t_{(1)}, \ldots, t_{(r)}$, $r \leq n$. The likelihood function is given by

$$
L(\mu, \sigma)=\left(\prod_{i=1}^{r} \frac{1}{\sigma_{(i)}} \phi\left(\frac{\ln t_{(i)}-\mu}{\sigma}\right)\left(Q\left(\frac{\ln t_{(r)}-\mu}{\sigma}\right)\right)^{n-r}\right.
$$

where $\phi()$ and $Q()$ are the probability density and the survival functions of the standard normal 
distribution. The likelihood function corresponding to Type 1 censoring is obtained by replacing $\ln t_{(r)}$ by $\ln t_{0}$, the censoring time under Type 1 censoring. The maximum likelihood estimator is obtained by finding $\hat{\mu}$ and $\hat{\sigma}$ that maximize the likelihood function. This is often done by equating the first partial derivatives of the log-likelihood function to zero and solving for $\mu$ and $\sigma$ simultaneously by applying an iterative numerical procedure for root finding like the Newton-Raphson method. However, this is problematic unless very good starting values are available (Lawless, 1982); the problem becomes serious when the proportion of censored observations is large, especially when the total sample size is relatively small to moderate. In such cases, alternatives to the maximum likelihood estimator are needed, either on their own or as initial approximations to the maximum likelihood estimators. The books of Lawless (1982), Schneider (1986) and Balakrishnan and Cohen (1991) survey much of the work in this area.

In this article, the performances of three techniques for point estimation of parameters in the case of censored data from a lognormal distribution will be extended, investigated, and compared. The first technique is based on finding the least squares estimator by regressing certain estimators of the linearized distribution function on a function of the observations themselves. This approach is used in Hossain and Howlader (1996) and Hossain and Zimmer (2003) for the parameters of the Weibull distribution. Their results showed that the estimators are a reasonable substitute for the maximum likelihood estimator in most situations.

The second technique is due to Perrson and Rootzen (1977) where they presented some modified likelihood function with Type 1 censored data whose maximizing point does not require iterative techniques. The last technique is based on expanding certain terms in the first derivatives of the log-likelihood function in an appropriate Taylor series to get a new system of likelihood equations whose solution exists in closed form. This last approach was studied for Type 2 censored data. An account of this work can be found in Balakrishnan and Cohen (1991).
Recently Al-Haj Ebarahem and Baklizi (2005) used the first and the last techniques to estimate the parameters of the Log-Logistic distribution based on complete and censored samples

Least Squares Estimators

The distribution function of the lognormal random variable is given by

$$
F(t)=\Phi\left(\frac{\ln t-\mu}{\sigma}\right)
$$

Linearization of this distribution function gives $\Phi^{-1}(F(t))=-\frac{\mu}{\sigma}+\frac{1}{\sigma} \ln t$.which is a linear regression model between $\Phi^{-1}(F(t))$ and $\ln t$. Let $T_{(1)}, \ldots, T_{(r)}$ be the observed censored sample and let $S_{i}$ be an estimate of $\Phi^{-1}\left(F\left(T_{(i)}\right)\right)$, then the least squares estimators of $b=\frac{1}{\sigma}$ and $a=-\frac{\mu}{\sigma}$ are given respectively by

$$
\hat{b}=\frac{\sum_{i=1}^{r} S_{i} \ln T_{i}-r \overline{\ln T} \bar{S}}{\sum_{i=1}^{r}\left(\ln T_{i}\right)^{2}-r(\overline{\ln T})^{2}}
$$

and

$$
\hat{a}=\bar{S}-\hat{b} \overline{\ln T}
$$

where

$$
\overline{\ln T}=\sum_{i=1}^{r} \ln T_{i} / r
$$

and

$$
\bar{S}=\sum_{i=1}^{r} S_{i} / r
$$


An estimate of $S_{i}, i=1, \ldots, r$ is now required.

Two methods of estimation of $F\left(T_{(i)}\right)$ and hence $S_{i}$ will be considered:

a) Let $\hat{F}\left(T_{(i)}\right)=1-R_{(i)}, i=1, \ldots, r$

where

$$
R_{(i)}=\frac{r_{i}}{r_{i}+1} R_{(i-1)}, R_{(0)}=1
$$

and

$$
r_{i}=n-r_{i}^{\prime}+1
$$

where $r_{i}^{\prime}$ is the rank of the $\mathrm{i}$-th failure in the original sample. Hence, $S_{i}=\Phi^{-1}\left(1-R_{(i)}\right)$. Substituting these values in $\hat{b}$ and $\hat{a}$, one obtains the estimators $\hat{\mu}_{1}$ and $\hat{\sigma}_{1}$.

b) Use $R_{(i)}=\frac{r_{i}-0.5}{r_{i-1}-0.5} R_{(i-1)}$. In this case the new least squares based estimators are based on $\hat{\mu}_{2}$ and $\hat{\sigma}_{2}$.

\section{Approximate Maximum Likelihood Estimators}

Let $T_{(1)} \leq T_{(2)} \leq \ldots \leq T_{(r)}$ be a Type 2 censored sample consisted of the smallest $r$ ordered observations obtained from the lognormal population with probability distribution function given by (1), the remaining $(n-r)$ observations being censored at $T_{(r)}$. Let $Y_{i}=\ln T_{(i)}, i=1, \ldots, r$ be the corresponding order statistics from the normal distribution. The likelihood function of $(\mu, \sigma)$ is given by equation (2). The maximum likelihood estimators $\hat{\mu}$ and $\hat{\sigma}$ of $\mu$ and $\sigma$ are given as the solution to the following simultaneous system of nonlinear equations (Lawless, 1982);

$$
\left.\frac{\partial \log L}{\partial \mu}=\frac{1}{\sigma^{2}} \sum_{i=1}^{r} y_{i}-\mu\right)+\frac{(n-r)}{\sigma}\left(\frac{\phi\left(\frac{y_{r}-\mu}{\sigma}\right)}{Q\left(\frac{y_{r}-\mu}{\sigma}\right)}\right)
$$

$$
\begin{aligned}
& \frac{\partial \log L}{\partial \sigma}=-\frac{r}{\sigma} \\
& +\frac{1}{\sigma^{3}} \sum_{i=1}^{r}\left(y_{i}-\mu\right)^{2}+\frac{(n-r)}{\sigma}\left(\frac{\frac{y_{r}-\mu}{\sigma} \phi\left(\frac{y_{r}-\mu}{\sigma}\right)}{Q\left(\frac{y_{r}-\mu}{\sigma}\right)}\right)
\end{aligned}
$$

The likelihood equations corresponding to Type 1 censoring are obtained by replacing $y_{r}=\ln t_{(r)}$ by $y_{0}=\ln t_{0}$, the censoring time under Type 1 censoring. As stated in the introduction, the system of equations (3) does not admit a closed form solution and a numerical method is needed to find the solution (the MLE). In the following two subsections, some modifications of these likelihood equations will be presented to obtain a closed form solution.

The Persson-Rootzen Approach

Consider the likelihood function (2) given by

$L(\mu, \sigma)=\left(\prod_{i=1}^{r} \frac{1}{\sigma} \phi\left(\frac{y_{(i)}-\mu}{\sigma}\right)\right)\left(Q\left(\frac{y_{(r)}-\mu}{\sigma}\right)\right)^{n-r}$

Putting

$$
x_{i}=y_{i}-y_{L}
$$

and

$$
\theta=\frac{y_{L}-\mu}{\sigma}
$$

where

$$
y_{L}= \begin{cases}\ln t_{0}, & \text { for type } 1 \text { censoring } \\ y_{(r)}, & \text { for type } 2 \text { censoring }\end{cases}
$$

where $t_{0}$ is the censoring time, write: 


$$
L(\mu, \sigma)=\frac{1}{\sigma^{r}} \exp \left(-\frac{1}{2 \sigma^{2}} \sum_{i=1}^{r}\left(x_{i}+\theta \sigma\right)^{2}\right) Q(\theta)^{n-r}
$$

Persson and Rootzen (1977) suggested replacing the survival function $Q(\theta)$ in (4) by its nonparameteric estimator $\frac{n-r}{n}$ and therefore replacing $\theta$ by $\theta^{*}=Q^{-1}\left(\frac{n-r}{n}\right)$, the $(r / n) t h$ quantile of the standard normal distribution. Substituting these quantities in (4), one obtains a function of $\sigma$ alone which is maximized by

$$
\hat{\sigma}_{3}=\frac{1}{2}\left(\frac{\theta^{*}}{r} \sum_{i=1}^{r} x_{i}+\left(\left(\frac{\theta^{*}}{r} \sum_{i=1}^{r} x_{i}\right)^{2}+\frac{4}{r} \sum_{i=1}^{r} x_{i}^{2}\right)^{1 / 2}\right)
$$

Substituting $\hat{\sigma}_{3}$ in (4) yields

$$
\hat{\mu}_{3}=y_{L}-\theta^{*} \hat{\sigma}_{3}
$$

Approximate MLE Based on Taylor Series Expansion

Consider the likelihood equations given

by (3)

$$
\frac{\partial \log L}{\partial \mu}=\frac{1}{\sigma^{2}} \sum_{i=1}^{r}\left(y_{i}-\mu\right)+\frac{(n-r)}{\sigma}\left(\frac{\phi\left(\frac{y_{r}-\mu}{\sigma}\right)}{Q\left(\frac{y_{r}-\mu}{\sigma}\right)}\right)
$$

$$
\begin{aligned}
& \frac{\partial \log L}{\partial \sigma}=-\frac{r}{\sigma} \\
& +\frac{1}{\sigma^{3}} \sum_{i=1}^{r}\left(y_{i}-\mu\right)^{2}+\frac{(n-r)}{\sigma}\left(\frac{\frac{y_{r}-\mu}{\sigma} \phi\left(\frac{y_{r}-\mu}{\sigma}\right)}{Q\left(\frac{y_{r}-\mu}{\sigma}\right)}\right)
\end{aligned}
$$

Let $Z_{i}=\frac{y_{i}-\mu}{\sigma}, i=1, \ldots, r$ and noting that $\phi^{\prime}(z)=-z \phi(z)$ obtains

$$
\begin{gathered}
\frac{\partial \log L}{\partial \mu}=\frac{1}{\sigma}\left(\sum_{i=1}^{r} z_{i}+(n-r) \frac{\phi\left(z_{r}\right)}{Q\left(z_{r}\right)}\right)=0 \\
\frac{\partial \log L}{\partial \sigma}=-\frac{1}{\sigma}\left(r-(n-r) z_{r} \frac{\phi\left(z_{r}\right)}{Q\left(z_{r}\right)}-\sum_{i=1}^{r} z_{i}^{2}\right)=0
\end{gathered}
$$

Expanding the function $\frac{\phi\left(z_{r}\right)}{Q\left(z_{r}\right)}$ in a Taylor series about the point $\xi_{r}=\Phi^{-1}\left(p_{r}\right)$, where $\Phi^{-1}($.$) is the inverse of the distribution function$ of the standard normal distribution and $p_{r}=\frac{r}{n+1} . \quad$ Setting $\quad q_{r}=1-p_{r} \quad$ obtains $\frac{\phi\left(z_{r}\right)}{Q\left(z_{r}\right)} \cong \gamma+\delta z_{r}$, where

and

$$
\gamma=\phi\left(\zeta_{r}\right)\left(1+\xi_{r}^{2}-\xi_{r} \phi\left(\zeta_{r}\right) / q_{r}\right) / q_{r}
$$

$$
\delta=\phi\left(\zeta_{r}\right)\left(\phi\left(\zeta_{r}\right)-q_{r} \xi_{r}\right) / q_{r}^{2}
$$

Substituting these quantities in the likelihood equations obtains

$$
\begin{aligned}
& \frac{\partial \log L}{\partial \mu} \cong \frac{1}{\sigma}\left(\sum_{i=1}^{r} z_{i}+(n-r) \gamma+(n-r) \delta z_{r}\right)=0 \\
& \frac{\partial \log L}{\partial \sigma} \cong-\frac{1}{\sigma}\left(r-(n-r) z_{r}-(n-r) \delta z_{r}^{2}-\sum_{i=1}^{r} z_{i}^{2}\right)=0
\end{aligned}
$$

Solving these equations yields the following:

$$
\begin{gathered}
\hat{\mu}_{4}=B-\hat{\sigma}_{4} C \\
\hat{\sigma}_{4}=\left(-D+\left(D^{2}+4 r E\right)^{1 / 2}\right) / 2 r,
\end{gathered}
$$

where

$$
B=\left(\sum_{i=1}^{r} y_{i}+(n-r) \delta y_{r}\right) / m,
$$




$$
\begin{gathered}
C=-(n-r) \gamma / m, \\
D=-(n-r) \gamma\left(y_{r}-B\right), \\
E=\sum_{i=1}^{r} y_{i}^{2}+(n-r) \delta y_{r}^{2}-m B^{2}
\end{gathered}
$$

and

$$
m=r+(n-r) \delta
$$

\section{Performance of the Estimators}

A simulation study is conducted to investigate the performance of the estimators. The simulation indices are the sample size $n=10,15,20,30,40,50,60,80,100,150$. The censoring proportion $c p: 0.1,0.3,0.5$, $a=1-c p$. For each combination of the simulation indices, 2,000 pairs of samples are generated and the maximum likelihood estimator $(\hat{\mu}, \hat{\sigma})$ and the closed form estimators $\left(\hat{\mu}_{i}, \hat{\sigma}_{i}\right), i=1 \ldots, 4$ are calculated. Their biases $B \hat{\mu}, B \hat{\sigma}$ and $B \hat{\mu}_{i}, B \hat{\sigma}_{i}, i=1, \ldots, 4$ and their mean squared errors and the relative efficiencies ef $\hat{\mu}_{i}=\frac{\operatorname{MSE}(\hat{\mu})}{\operatorname{MSE}\left(\hat{\mu}_{i}\right)} \quad$ and $\quad e f \hat{\sigma}_{i}=\frac{\operatorname{MSE}(\hat{\sigma})}{\operatorname{MSE}\left(\hat{\sigma}_{i}\right)}$ $, i=1 \ldots, 4$ are obtained.

\section{Results}

The results are given in Tables $1-4$. The biases of the estimators are given in Tables $1-2$ and the efficiencies of the estimators are given in tables $3-4$. Inspection of the simulation numerical results lead to the following observations and conclusions. It appears that, under Type 1 censoring, $\hat{\mu}_{1}$ and $\hat{\mu}_{2}$ are positively biased when the censoring proportion is moderate to heavy. This is true for all sample sizes. In all other cases, all estimators tend to be negatively biased, regardless of the sample size. It appears that $\hat{\mu}_{3}$ has the highest bias, and the least bias is achieved by $\hat{\mu}_{3}$ for light censoring and $\hat{\mu}_{2}$ and $\hat{\mu}_{5}$ for moderate to heavy censoring.

For estimators of the scale parameter $\sigma$ under Type 1 censoring, it appears that $\hat{\sigma}$ has the least bias followed by $\hat{\sigma}_{3}$ and $\hat{\sigma}_{4}$. The performances of $\hat{\sigma}_{3}$ and $\hat{\sigma}_{4}$ in terms of bias is about similar. However, $\hat{\sigma}_{1}$ tends to have the largest bias among the estimators considered.

The relative performance of estimators under Type 2 censoring is similar to that of Type 1 censoring. In all cases, the bias decreases as the sample size increases. It is also smaller for lighter censoring.

Concerning the relative efficiencies of the estimators under Type 1 censoring, it appears that the following schemes hold, $\hat{\mu}_{4}>\hat{\mu}_{3}>\hat{\mu}_{2}>\hat{\mu}_{1}$ under heavy censoring regardless of the sample size and $\hat{\mu}_{4}>\hat{\mu}_{1}>\hat{\mu}_{2}>\hat{\mu}_{3}$ for moderate to light censoring, where $(>)$ means more efficient. It also appears that the relative efficiencies of $\hat{\mu}_{1}, \hat{\mu}_{2}$ and $\hat{\mu}_{3}$ do not depend on the sample size. However, the relative efficiency of $\hat{\mu}_{4}$ increases as sample size increases. The relative efficiencies of $\hat{\mu}_{2}$ and $\hat{\mu}_{3}$ increase as the censoring proportion becomes smaller, while it decreases for $\hat{\mu}_{4}$.

The results show that, under Type 1 censoring $\hat{\mu}_{4}$ are more efficient than the MLE. With regard to scale estimators under Type 1 censoring, it appears that $\hat{\sigma}_{4}>\hat{\sigma}_{3}>\hat{\sigma}_{2}>\hat{\sigma}_{1}$, whereas before $(>)$ indicated more efficient. It appears that the relative efficiencies of the scale estimators do not depend on $n$; however, they depend on the censoring proportion. As the censoring proportion becomes smaller, the relative efficiencies of $\hat{\sigma}_{1}, \hat{\sigma}_{2}$ and $\hat{\sigma}_{4}$ increases and it decreases for $\hat{\sigma}_{3}$. Surprisingly, in all cases considered, the approximate estimators $\hat{\sigma}_{4}$ are more efficient than the corresponding MLE. 
176 COMPARISON OF SIMPLE ESTIMATORS OF LOGNORMAL PARAMETERS

Table 1. Bias of the Estimators Under Type 1 Censoring

\begin{tabular}{|c|c|c|c|c|c|c|c|c|c|c|c|}
\hline$n$ & $a$ & $B \hat{\mu}_{1}$ & $B \hat{\mu}_{2}$ & $B \hat{\mu}_{3}$ & $B \hat{\mu}_{4}$ & $B \hat{\mu}$ & $B \hat{\sigma}_{1}$ & $B \hat{\sigma}_{2}$ & $B \hat{\sigma}_{3}$ & $B \hat{\sigma}_{4}$ & $B \hat{\sigma}$ \\
\hline 10 & 0.5 & 0.106 & 0.039 & -0.121 & -0.114 & -0.099 & 0.268 & 0.239 & -0.194 & -0.195 & -0.188 \\
\hline 10 & 0.7 & 040 & -0.033 & -0.083 & -0.055 & -0.041 & 0.231 & 0.193 & -0.141 & -0.138 & -0.125 \\
\hline 10 & 0.9 & -0.000 & -0.086 & -0.103 & -0.016 & -0.015 & 0.191 & 0.131 & -0.149 & -0.108 & -0.099 \\
\hline 15 & 0.5 & 0.088 & 0.043 & -0.075 & -0.066 & -0.056 & 0.221 & 0.203 & -0.118 & -0.118 & -0.112 \\
\hline 15 & 0.7 & 0.030 & -0.019 & -0.047 & -0.032 & -0.018 & 0.175 & 0.149 & -0.085 & -0.086 & -0.073 \\
\hline 15 & 0.9 & -0.007 & -0.069 & -0.103 & -0.010 & -0.014 & 0.153 & 0.107 & -0.115 & -0.062 & -0.057 \\
\hline 20 & 0.5 & 0.079 & 0.047 & -0.062 & -0.059 & -0.051 & 0.184 & 0.171 & -0.094 & -0.096 & -0.091 \\
\hline 20 & 0.7 & 0.027 & -0.008 & -0.041 & -0.028 & & 0.139 & 122 & -0.074 & -0.074 & -0.066 \\
\hline 20 & 0.9 & 0.005 & -0.038 & -0.050 & -0.009 & -0 & 0.126 & .096 & -0.069 & -0.055 & -0.041 \\
\hline 30 & 0.5 & 0.078 & 0.057 & -0.036 & -0.033 & -0.026 & 0.147 & 0.139 & -0.063 & -0.064 & -0.061 \\
\hline 30 & 0.7 & 0.025 & 0.001 & -0.024 & -0.018 & & 0.108 & 0.096 & -0.046 & -0.049 & -0 \\
\hline 30 & 0.9 & 0.007 & -0.021 & -0.031 & -0.007 & & 0.098 & .079 & -0.041 & -0.035 & -0. \\
\hline 40 & 0.5 & 0.051 & 0.036 & -0.039 & -0.033 & -0. & 0.117 & 0.111 & -0.050 & -0.050 & -0.049 \\
\hline 40 & 0.7 & 0.013 & -0.004 & -0.026 & -0.019 & -0. & 0.089 & 0.081 & .033 & -0.033 & -0.029 \\
\hline 40 & 0.9 & -0. & -0.022 & -0. & & & 0.071 & 057 & 038 & -0.030 & 22 \\
\hline 50 & 0.5 & 0.050 & 0.038 & -0.030 & -0.025 & -0.02 & 0.102 & 0.097 & -0.041 & -0.041 & -0.040 \\
\hline 50 & 0.7 & 0.015 & 0.001 & -0.020 & -0.013 & -0.0 & 0.079 & 0.072 & -0.025 & -0.025 & -0.022 \\
\hline 50 & 0.9 & 0. & -0.014 & -0.022 & -0 . & & 0.066 & 0.054 & -0.026 & -0.021 & -0.012 \\
\hline 60 & 0.5 & 0.051 & 0.041 & -0.022 & -0.019 & -0.016 & 0.103 & 0.099 & -0.024 & -0.024 & -0.022 \\
\hline 60 & 0.7 & 0.013 & 0.002 & -0.014 & -0.011 & -0.00 & 0.065 & 0.060 & -0.023 & -0.024 & -0.020 \\
\hline 60 & 0.9 & 0.001 & -0.012 & -0.019 & -0.005 & -0. & 0.053 & 0.044 & -0.025 & -0.020 & -0.014 \\
\hline 80 & 0.5 & 0.035 & 0.027 & -0.019 & -0.016 & -0.016 & 0.076 & 0.074 & -0.020 & -0.020 & -0.019 \\
\hline 80 & 0.7 & 0.01 & 0.006 & -0.008 & -0.005 & -0.0 & 0.050 & 0.047 & -0.019 & -0.020 & -0.017 \\
\hline 80 & 0.9 & -0.002 & -0.012 & -0.016 & -0.004 & -0.003 & 0.036 & 0.029 & -0.022 & -0.019 & -0.015 \\
\hline 100 & 0.5 & 0.034 & 0.028 & -0.014 & -0.012 & -0.011 & 0.069 & 0.067 & -0.014 & -0.015 & -0.014 \\
\hline 100 & 0.7 & 0.009 & 0.003 & -0.010 & -0.007 & -0.005 & 0.048 & 0.045 & -0.011 & -0.011 & -0.009 \\
\hline 100 & 0.9 & -0.001 & -0.010 & -0.014 & -0.006 & -0.002 & 0.036 & 0.030 & -0.013 & -0.012 & -0.007 \\
\hline 150 & 0.5 & 0.026 & 0.022 & -0.008 & -0.007 & -0.006 & 0.048 & 0.046 & -0.011 & -0.012 & -0.011 \\
\hline 150 & 0.7 & 0.005 & 0.001 & -0.009 & -0.005 & -0.005 & 0.035 & 0.033 & -0.008 & -0.007 & -0.006 \\
\hline 150 & 0.9 & -0.001 & -0.006 & -0.008 & -0.004 & -0.001 & 0.025 & 0.022 & -0.008 & -0.008 & -0.004 \\
\hline
\end{tabular}


Table 2. Bias of the Estimators Under Type 2 Censoring

\begin{tabular}{|c|c|c|c|c|c|c|c|c|c|c|c|}
\hline$n$ & $a$ & $B \hat{\mu}_{1}$ & $B \hat{\mu}_{2}$ & $B \hat{\mu}_{3}$ & $B \hat{\mu}_{4}$ & $B \hat{\mu}$ & $B \hat{\sigma}_{1}$ & $B \hat{\sigma}_{2}$ & $B \hat{\sigma}_{3}$ & $B \hat{\sigma}_{4}$ & $B \hat{\sigma}$ \\
\hline 10 & 0.5 & 0.117 & 0.049 & -0.114 & -0.092 & -0.091 & 0.285 & 0.256 & -0.185 & -0.178 & -0.178 \\
\hline 10 & 0.7 & 050 & .023 & -0.064 & -0.028 & -0.027 & 0.221 & 0.184 & -0.141 & -0.128 & -0.127 \\
\hline 10 & 0.9 & -0.000 & -0.086 & -0.108 & -0.016 & -0.015 & 0.201 & 0.140 & -0.145 & -0.094 & -0.093 \\
\hline 15 & 0.5 & 0.100 & 0.057 & -0.109 & -0.093 & -0.092 & 0.231 & 0.214 & -0.143 & -0.138 & -0.138 \\
\hline 15 & 0.7 & 0.059 & 0.011 & -0.040 & -0.014 & -0.014 & 0.193 & 0.170 & -0.089 & -0.081 & -0.080 \\
\hline 15 & 0.9 & 0.007 & -0.048 & -0.064 & -0.009 & -0.009 & 0.160 & 124 & -0.087 & -0.059 & -0.059 \\
\hline 20 & 0.5 & 0.088 & 0.056 & -0.060 & -0.048 & -0. & 0.192 & 0.180 & -0.092 & -0.089 & -0.089 \\
\hline 20 & 0.7 & 34 & -0.001 & -0.032 & -0.013 & & 0.140 & 122 & 072 & 65 & 65 \\
\hline 20 & 0.9 & 007 & -0.036 & -0.048 & 0.000 & & 0.123 & 094 & -0.071 & & \\
\hline 30 & 0.5 & 0.078 & 0.057 & -0.039 & -0.029 & -0.029 & 0.149 & 0.141 & -0.065 & -0.062 & -0.062 \\
\hline 30 & 0.7 & 027 & 0.003 & -0.025 & -0.010 & & 0.115 & 104 & 043 & & 37 \\
\hline 30 & 0.9 & 0.002 & -0.026 & -0.034 & -0.001 & -0. & .084 & 065 & & & 333 \\
\hline 40 & 0.5 & 0.063 & 0.047 & -0.034 & -0.026 & -0. & 0.123 & 0.117 & -0.049 & -0 & -0.047 \\
\hline 40 & 0.7 & 0.022 & 0.005 & -0.018 & -0.007 & -0.007 & 0.089 & 081 & -0.035 & 30 & -0.030 \\
\hline 40 & 0.9 & 04 & -0 . & -0.025 & 0.001 & & .069 & 55 & & & \\
\hline 50 & 0.5 & 0.047 & 0.035 & -0.035 & -0.028 & -0.028 & 0.101 & 0.097 & -0.043 & -0.042 & -0.041 \\
\hline 50 & 0.7 & 020 & 0.007 & -0.013 & -0.004 & -0.004 & 0.076 & 0.069 & -0.027 & -0.024 & -0.024 \\
\hline 50 & 0.9 & -0.000 & -0.017 & -0.024 & -0.002 & -0 . & 0.061 & 0.050 & -0.029 & & 016 \\
\hline 60 & 0.5 & 0.041 & 0.031 & -0.027 & -0.023 & -0.023 & 0.090 & 0.086 & -0.033 & -0.032 & -0.032 \\
\hline 60 & 0.7 & 0.019 & 0.007 & -0.014 & -0.004 & -0.0 & 0.067 & 0.061 & -0.025 & 22 & -0.022 \\
\hline 60 & 0.9 & -0.001 & -0.015 & -0.020 & -0.003 & -0.003 & 0.053 & 0.043 & -0.023 & -0.013 & -0.013 \\
\hline 80 & 0.5 & 0.040 & 0.033 & -0.014 & -0.011 & -0.011 & 0.076 & 0.073 & -0.022 & -0.021 & -0.021 \\
\hline 80 & 0.7 & 0.011 & 0.002 & -0.01 & -0.006 & -0.006 & 0.054 & 0.050 & -0.016 & & -0.014 \\
\hline 80 & 0.9 & 0.001 & -0.009 & -0.015 & -0.001 & -0.001 & 0.039 & 0.032 & -0.022 & -0.013 & -0.013 \\
\hline 100 & 0.5 & 0.034 & 0.028 & -0.012 & -0.009 & -0.009 & 0.060 & 0.058 & -0.022 & -0.021 & -0.021 \\
\hline 100 & 0.7 & 0.016 & 0.009 & -0.005 & 0.000 & & 0.048 & 0.045 & -0.012 & -0.010 & -0.010 \\
\hline 100 & 0.9 & 0.002 & -0.005 & -0.009 & 0.001 & 0. & 0.035 & 0.030 & -0.014 & -0.008 & -0.008 \\
\hline 150 & 0.5 & 0.028 & 0.024 & -0.007 & -0.005 & -0.005 & 0.050 & 0.049 & -0.010 & -0.009 & -0.009 \\
\hline 150 & 0.7 & 0.010 & 0.005 & -0.004 & -0.001 & -0.001 & 0.031 & 0.029 & -0.010 & -0.009 & -0.009 \\
\hline 150 & 0.9 & 0.001 & -0.004 & -0.006 & 0.001 & 0.0 & 0.026 & 0.022 & -0.008 & -0.004 & -0.004 \\
\hline
\end{tabular}


Table 3. Efficiencies of the Estimators Under Type 1 Censoring

\begin{tabular}{|c|c|c|c|c|c|c|c|c|c|}
\hline$n$ & $a$ & $e f \hat{\mu}_{1}$ & $e f \hat{\mu}_{2}$ & $e f \hat{\mu}_{3}$ & $e f \hat{\mu}_{4}$ & $e f \hat{\sigma}_{1}$ & $e f \hat{\sigma}_{2}$ & $e f \hat{\sigma}_{3}$ & $e f \hat{\sigma}_{4}$ \\
\hline 10 & 0.5 & 0.741 & 0.835 & 0.977 & 1.718 & 0.388 & 0.419 & 0.994 & 1.054 \\
\hline 10 & 0.7 & 0.917 & 0.957 & 0.932 & 2.095 & 0.421 & 0.477 & 0.975 & 1.143 \\
\hline 10 & 0.9 & 1.000 & 0.937 & 0.822 & 1.563 & 0.472 & 0.600 & 0.863 & 1.200 \\
\hline 15 & 0.5 & 0.745 & 0.811 & 0.981 & 2.109 & 0.399 & 0.425 & 0.991 & 1.097 \\
\hline 15 & 0.7 & 0.930 & 0.952 & 0.927 & 2.208 & 0.459 & 0.510 & 0.964 & 1.180 \\
\hline 15 & 0.9 & 0.999 & 0.935 & 0.755 & 1.438 & 0.495 & 0.623 & 0.792 & 1.278 \\
\hline 20 & 0.5 & 0.732 & 0.787 & 0.966 & 2.320 & 0.446 & 0.467 & 0.989 & 1.098 \\
\hline 20 & 0.7 & 0.891 & 0.915 & 0.936 & 2.373 & 0.496 & 0.537 & 0.959 & 1.179 \\
\hline 20 & 0.9 & 0.997 & 0.971 & 0.810 & 1.485 & 0.535 & 0.626 & 0.856 & 1.293 \\
\hline 30 & 0.5 & 0.674 & 0.714 & 0.989 & 2.521 & 0.439 & 0.454 & 1.000 & 1.100 \\
\hline 30 & 0.7 & 0.878 & 0.902 & 0.939 & 2.520 & 0.534 & 0.565 & 0.971 & 1.243 \\
\hline 30 & 0.9 & 0.983 & 0.973 & 0.832 & 1.438 & 0.551 & 0.625 & 0.855 & 1.335 \\
\hline 40 & 0.5 & 0.736 & 0.767 & 0.966 & 2.727 & 0.489 & 0.503 & 0.993 & 1.126 \\
\hline 40 & 0.7 & 0.897 & 0.910 & 0.925 & 2.753 & 0.548 & 0.575 & 0.968 & 1.291 \\
\hline 40 & 0.9 & 0.989 & 0.973 & 0.814 & 1.494 & 0.635 & 0.701 & 0.837 & 1.377 \\
\hline 50 & 0.5 & 0.725 & 0.752 & 0.973 & 2.847 & 0.512 & 0.524 & 0.994 & 1.132 \\
\hline 50 & 0.7 & 0.890 & 0.905 & 0.930 & 2.827 & 0.571 & 0.594 & 0.972 & 1.291 \\
\hline 50 & 0.9 & 0.986 & 0.978 & 0.813 & 1.505 & 0.613 & 0.670 & 0.852 & 1.358 \\
\hline 60 & 0.5 & 0.707 & 0.734 & 0.970 & 3.018 & 0.490 & 0.501 & 0.992 & 1.145 \\
\hline 60 & 0.7 & 0.884 & 0.898 & 0.935 & 2.867 & 0.601 & 0.624 & 0.963 & 1.306 \\
\hline 60 & 0.9 & 0.991 & 0.982 & 0.804 & 1.528 & 0.663 & 0.715 & 0.859 & 1.354 \\
\hline 80 & 0.5 & 0.712 & 0.730 & 0.977 & 3.119 & 0.518 & 0.528 & 0.993 & 1.145 \\
\hline 80 & 0.7 & 0.910 & 0.924 & 0.911 & 3.171 & 0.625 & 0.643 & 0.969 & 1.277 \\
\hline 80 & 0.9 & 0.991 & 0.980 & 0.801 & 1.571 & 0.754 & 0.798 & 0.836 & 1.447 \\
\hline 100 & 0.5 & 0.702 & 0.718 & 0.975 & 3.224 & 0.532 & 0.541 & 0.993 & 1.145 \\
\hline 100 & 0.7 & 0.901 & 0.911 & 0.919 & 3.152 & 0.616 & 0.632 & 0.975 & 1.307 \\
\hline 100 & 0.9 & 0.988 & 0.978 & 0.801 & 1.482 & 0.725 & 0.764 & 0.821 & 1.437 \\
\hline 150 & 0.5 & 0.719 & 0.733 & 0.972 & 3.309 & 0.588 & 0.595 & 0.998 & 1.158 \\
\hline 150 & 0.7 & 0.913 & 0.918 & 0.923 & 3.307 & 0.677 & 0.691 & 0.956 & 1.351 \\
\hline 150 & 0.9 & 0.988 & 0.983 & 0.806 & 1.528 & 0.758 & 0.789 & 0.833 & 1.436 \\
\hline
\end{tabular}


Table 4. Efficiencies of the Estimators Under Type 2 Censoring

\begin{tabular}{|l|l|l|l|l|l|l|l|l|l|}
\hline$n$ & $a$ & $e f \hat{\mu}_{1}$ & $e f \hat{\mu}_{2}$ & $e f \hat{\mu}_{3}$ & $e f \hat{\mu}_{4}$ & $e f \hat{\sigma}_{1}$ & $e f \hat{\sigma}_{2}$ & $e f \hat{\sigma}_{3}$ & $e f \hat{\sigma}_{4}$ \\
\hline 10 & 0.5 & 0.723 & 0.821 & 0.978 & 0.999 & 0.370 & 0.400 & 0.992 & 0.999 \\
\hline 10 & 0.7 & 0.921 & 0.972 & 0.929 & 1.000 & 0.445 & 0.505 & 0.961 & 0.999 \\
\hline 10 & 0.9 & 0.999 & 0.934 & 0.807 & 0.999 & 0.452 & 0.577 & 0.869 & 0.999 \\
\hline 15 & 0.5 & 0.688 & 0.753 & 0.980 & 0.999 & 0.395 & 0.416 & 0.996 & 0.999 \\
\hline 15 & 0.7 & 0.853 & 0.910 & 0.954 & 0.999 & 0.425 & 0.463 & 0.980 & 0.999 \\
\hline 15 & 0.9 & 0.978 & 0.950 & 0.866 & 0.999 & 0.487 & 0.575 & 0.900 & 0.999 \\
\hline 20 & 0.5 & 0.709 & 0.764 & 0.975 & 0.999 & 0.429 & 0.449 & 0.992 & 0.999 \\
\hline 20 & 0.7 & 0.908 & 0.939 & 0.917 & 1.000 & 0.507 & 0.547 & 0.963 & 0.999 \\
\hline 20 & 0.9 & 0.982 & 0.961 & 0.856 & 1.000 & 0.531 & 0.622 & 0.842 & 1.000 \\
\hline 30 & 0.5 & 0.693 & 0.733 & 0.974 & 0.999 & 0.439 & 0.454 & 0.996 & 0.999 \\
\hline 30 & 0.7 & 0.880 & 0.907 & 0.919 & 1.000 & 0.499 & 0.529 & 0.975 & 0.999 \\
\hline 30 & 0.9 & 0.990 & 0.970 & 0.814 & 1.000 & 0.621 & 0.698 & 0.851 & 0.999 \\
\hline 40 & 0.5 & 0.687 & 0.720 & 0.982 & 0.999 & 0.455 & 0.468 & 1.001 & 0.999 \\
\hline 40 & 0.7 & 0.896 & 0.919 & 0.919 & 1.000 & 0.549 & 0.576 & 0.971 & 0.999 \\
\hline 40 & 0.9 & 0.986 & 0.976 & 0.825 & 1.000 & 0.639 & 0.703 & 0.864 & 0.999 \\
\hline 50 & 0.5 & 0.700 & 0.725 & 0.978 & 0.999 & 0.503 & 0.515 & 0.991 & 1.000 \\
\hline 50 & 0.7 & 0.890 & 0.909 & 0.936 & 1.000 & 0.572 & 0.595 & 0.974 & 1.000 \\
\hline 50 & 0.9 & 0.992 & 0.977 & 0.796 & 1.000 & 0.652 & 0.710 & 0.846 & 0.999 \\
\hline 60 & 0.5 & 0.716 & 0.738 & 0.977 & 0.999 & 0.492 & 0.502 & 1.001 & 0.999 \\
\hline 60 & 0.7 & 0.882 & 0.900 & 0.926 & 0.999 & 0.590 & 0.611 & 0.972 & 0.999 \\
\hline 60 & 0.9 & 0.994 & 0.981 & 0.795 & 1.000 & 0.670 & 0.722 & 0.847 & 1.000 \\
\hline 80 & 0.5 & 0.709 & 0.729 & 0.970 & 1.000 & 0.525 & 0.534 & 0.995 & 1.000 \\
\hline 80 & 0.7 & 0.903 & 0.915 & 0.912 & 1.000 & 0.610 & 0.629 & 0.968 & 1.000 \\
\hline 80 & 0.9 & 0.986 & 0.981 & 0.833 & 1.000 & 0.722 & 0.765 & 0.844 & 0.999 \\
\hline 100 & 0.5 & 0.728 & 0.745 & 0.974 & 1.000 & 0.572 & 0.581 & 0.994 & 1.000 \\
\hline 100 & 0.7 & 0.902 & 0.917 & 0.917 & 1.000 & 0.616 & 0.633 & 0.973 & 1.000 \\
\hline 100 & 0.9 & 0.987 & 0.985 & 0.815 & 1.000 & 0.725 & 0.765 & 0.830 & 1.000 \\
\hline 150 & 0.5 & 0.713 & 0.726 & 0.981 & 1.000 & 0.573 & 0.580 & 0.998 & 1.000 \\
\hline 150 & 0.7 & 0.912 & 0.921 & 0.918 & 1.000 & 0.689 & 0.701 & 0.967 & 1.000 \\
\hline 150 & 0.9 & 0.988 & 0.986 & 0.823 & 1.000 & 0.751 & 0.780 & 0.865 & 1.000 \\
\hline & & & & & & & & & \\
\hline
\end{tabular}


The performance of the estimators under Type 2 censoring is similar to their performance under Type 1 censoring. However it appears that $\hat{\sigma}_{3}$ and $\hat{\sigma}_{4}$ are about as efficient as the MLE for all sample sizes and censoring proportions, except for $\hat{\sigma}_{3}$ when the censoring proportion is small, in which case $\hat{\sigma}_{3}$ is less efficient.

\section{Conclusion}

It appears that good substitutes to the MLE in closed form do exist. The performance of some of them is highly competent with that of the MLE and sometimes they are better, as is the case with the approximation based on the Taylor series expansion $\hat{\mu}_{4}$ and $\hat{\sigma}_{4}$.

\section{References}

Balakrishnan \& Cohen (1991). Order statistics and inference. Chapman and Hall.

Hossain, A. \& Howlader H. A. (1996). Unweighted least squares estimation of Weibull parameters. Journal of Statistical Computation and Simulation, 54, 265-271.

Hossain, M., Anwar, \& Zimmer, J. W. (2003). Comparison of estimation methods for Weibull parameters: Complete and censored samples. Journal of Statistical Computation and Simulation, 73(2), 145-153
Johnson, N. L, Kotz, S., \& Balakrishnan. (1994). Continuous univariate distributions (vol. 1). New York: Wiley.

Koch, A. L. (1966). The logarithm in biology. Journal of Theoretical Biology, 12, 276-290.

Lawless, J. F. (1982). Statistical models and methods for lifetime data. NewYork: Wiley.

Lyles, R. H., Kupper, L. L., \& Rappaport, S. M. (1997). Assessing regulatory compliance of occupational exposures via the balanced one-way random effects ANOVA model. Journal of Agricultural, Biological, and Environmental Statistics, 2, 64-86.

Ebrahem, M. A. \& Baklizi, A. (2005). Comparison of some simple estimators of the log-logistic parameters based on complete and censored samples. Journal of Statistical Theory and Applications, 4(2): $151-160$.

Persson, T. \& Rootzen, H. (1977). Simple and highly efficient estimators for a type 1 censored normal sample. Biometrika, 64, 123128.

Schneider, H. (1986). Truncated and censored samples from normal populations. New York: Marcel Dekker. 